Revista de

Contabilidade e

Organizações

www.rco.usp.br
DOI: http://dx.doi.org/10.11606/rco.v9i23.61873
Journal of

Accounting and

Organizations

\title{
Valor de mercado e valor contábil e sua relação com os resultados anormais no mercado de capitais no Brasil
}

\author{
Dimitri Pinheiro de Sant'Anna ${ }^{\mathrm{a}}$; Luiz Cláudio Louzada ${ }^{\mathrm{b}}$; Edson Queiroz ${ }^{\mathrm{c}}$; Bruno Pérez Ferreira ${ }^{\mathrm{d}}$ \\ ${ }^{a}$ Fundação Instituto Capixaba de Pesquisas em Contabilidade, Economia e Finanças - Fucape \\ ${ }^{b}$ Universidade Federal de Minas Gerais \\ c Fundação Instituto Capixaba de Pesquisas em Contabilidade, Economia e Finanças - Fucape \\ ${ }^{d}$ Universidade Federal de Minas Gerais
}

\section{Informações do Artigo}

\section{Histórico do Artigo}

Recebido: 11 de setembro de 2013

Aceito: 10 de janeiro de 2014

Palavras chave:

Resultado Anormal

Modelo de Ohlson

Price/Book

\begin{abstract}
Resumo
Este estudo analisa a relação entre o Price/Book (P/B) das ações negociadas na Bolsa de Valores e os Resultados Anormais. O conceito de Resultado Anormal (residual income) utilizado neste estudo baseia-se no Modelo de Residual Income Valuation de Ohlson e fornece o arcabouço teórico para se valorizar/avaliar as empresas a partir de seus resultados contábeis, relacionando, consequentemente, os resultados anormais com o Price/Book apresentado pelas ações das empresas. Testou-se, a partir de duas carteiras de ações (uma com os maiores $\mathrm{P} / \mathrm{B}$ e outra com os menores $\mathrm{P} / \mathrm{B}$ para cada ano entre 1996 e 2014), se as empresas que apresentavam os maiores P/B seriam aquelas empresas que geravam os maiores resultados anormais. Os testes indicaram que realmente as carteiras de maior $\mathrm{P} / \mathrm{B}$ foram as que apresentaram os maiores resultados anormais, pelo menos para dezesseis dos dezenove anos pesquisados, já que para três anos os resultados não puderam ser considerados estatisticamente diferentes a um nível de significância de cinco por cento.
\end{abstract}

Copyright (C) 2015 FEA-RP/USP. Todos os direitos reservados

\section{INTRODUÇÃO}

Este trabalho estuda o indicador Price/Book (ou Preço da Ação/Valor Patrimonial por ação) das ações negociadas na Bolsa de Valores de São Paulo e sua relação com os Resultados Anormais gerados pelas respectivas empresas. O Price/Book (também conhecido como Market-to-Book Equity, ME/BE) é a razão (ratio) entre o valor de mercado de uma empresa e o valor patrimonial da mesma expresso em seus livros/relatórios contábeis. O Price/Book indica como o mercado está valorizando a empresa em relação aos seus dados contábeis. Quando esta proporção é maior do que 1 (um) significa que o mercado valoriza determinada empresa mais do que está registrado/calculado nos seus livros e/ou relatórios contábeis, ou seja, o mercado está reconhecendo algo que não está sendo registrado/captado pela contabilidade (ou que está sendo feito apenas parcialmente). E quando esta proporção é menor que 1 (um) significa que o mercado valoriza determinada empresa por menos do que está registrado em seus livros contábeis, ou seja, o mercado não está reconhecendo, total ou parcialmente, valores que a contabilidade está registrando em seus livros.

Os Resultados Anormais (ou comumente chamados de Lucros Anormais) são os resultados líquidos (ou residuais) que uma empresa obtém após descontar, de seus resultados, os valores que seriam devidos pela aplicação de seu Capital a uma determinada taxa mínima de atratividade ou rentabilidade. Tradicionalmente se utiliza uma taxa baseada no Custo de Capital da empresa para se chegar aos resultados anormais obtidos no período, entretanto, este trabalho, baseado no arcabouço teórico do Modelo de Ohlson (Residual Income Valuation), utiliza a taxa livre de risco para calcular os resultados anormais da empresa.

Autor Correspondente: Tel (27) 40092602

E-mail : dimitri.santanna@arcelormittal.com.br(D. Sant'Anna); louzadalvi@yahoo.com.br (L. C. Louzada); edsonqueiroz_3@hotmail.com (E. Queiroz); brunoperez.bh@gmail.com (B. P. Ferreira)

Universidade Federal do Espírito Santo, Centro de Ciências Jurídicas e Econômicas - Av. Fernando Ferrari, 514 - Goiabeiras - 29075910 - Vitória, ES - Brasil. 
James Ohlson desenvolveu um modelo de avaliação de empresas chamado de Residual Income Valuation (RIV) no qual os lucros (e não os dividendos distribuídos) são a base para se calcular o valor da empresa.

A partir desta proposta de Ohlson, cabe então avaliar: se o Price/Book representa a relação entre o valor da empresa reconhecido pelo mercado e o valor contábil da empresa e, se o valor da empresa pode ser calculado a partir de seus resultados, então as empresas que expressam os maiores índices de Price/Book (ou maiores ME/BE) devem ser aquelas empresas que o mercado acredita que estejam/estarão auferindo os maiores resultados.

\section{O mercado de capitais no Brasil, com todas as suas especificidades, estaria avaliando as empresas desta maneira e/ou de acordo com estas premissas?}

Para responder a esta pergunta estudou-se as relações entre estas variáveis apresentadas pelas empresas/ ações negociadas na Bolsa de Valores de São Paulo entre os anos de 1996 e 2014, a partir da separação/classificação das ações em duas carteiras: uma com as ações que apresentavam os maiores Price/Book (ME/BE) e outra com os menores Price/Book (ME/BE) a fim de se verificar se a primeira apresentava maiores resultados anormais do que a segunda.

Na literatura de finanças, diversos autores (LAKONISHOK, SHLEIFER e VISHNY, 1994; PENMAN, 1996; FAMA e FRENCH) se utilizaram metodologicamente do estudo de carteiras (portfólios) para testar relações entre variáveis. Os artigos de Fama e French $(1992,1995,1996)$ servem de referencial tanto metodológico como teórico para este trabalho, já que pesquisaram o efeito tamanho (size effect) e o Market-to-Book (Price/Book) e suas relações com o retorno das ações e com os lucros das empresas. Os trabalhos de Fama e French $(1992,1995,1996)$ estudaram períodos bem maiores (trinta anos em alguns casos), amostras bem maiores (mais de quatro mil ações) e, ainda que não tratassem de resultados anormais, muitas de suas premissas e constatações foram de grande valia para este trabalho.

Espera-se, com este estudo, contribuir para o aprimoramento das relações entre a Contabilidade e o Mercado de Capitais no Brasil. As próximas seções deste estudo abordam os Resultados Anormais; a relação Price/Book; a hipótese do trabalho; a metodologia; os resultados; e as considerações finais.

\section{RESULTADOS ANORMAIS}

O conceito de Resultado Anormal (residual income) utilizado neste estudo baseia-se no Modelo de Residual Income Valuation de Ohlson (1995) e é apurado do seguinte modo: o Resultado Anormal do período é calculado pela diferença entre o Resultado Contábil verificado neste mesmo período e o produto do Patrimônio Líquido do período anterior pela Taxa livre de Risco do período. Ou seja, deduz-se do Resultado Contábil a parcela que seria correspondente à aplicação do PL a uma taxa de juros livre de risco. Conforme proposto por Victor Bernard (LOPES, 2001, p. 155):

$$
A b_{i j}=R C_{i j}-\left(B V_{i j-1} * R F_{j}\right)
$$

Onde:

$\boldsymbol{A} \boldsymbol{b}_{i j} \quad$ é o Resultado anormal contábil por ação da empresa i no período j

$\boldsymbol{R} \boldsymbol{C}_{i j}$ é o Resultado contábil por ação da empresa i registrado no período $\mathrm{j}$

$\boldsymbol{B} \boldsymbol{V}_{i j-1}$ é o Valor Patrimonial (PL) por ação da empresa i registrado no período j-1

$\boldsymbol{R} \boldsymbol{F}$ é a Taxa de juros livre de risco no período j

Lopes (2001, p. 156) ressalta que "este conceito de resultado anormal não é o mesmo de residual income tradicionalmente utilizado na literatura. A taxa de juros livre de risco é utilizada neste modelo ao invés do custo de capital da empresa utilizado em modelos de residual income".

Seguindo-se o modelo proposto por Ohlson, também se adotou uma taxa de juros livre de risco para se calcular os Resultados Anormais das empresas neste estudo. Optou-se pela poupança para servir como taxa livre de risco no Brasil durante este período, já que, conforme Securato (1996, p. 128), 
Na economia brasileira poderíamos considerar como risco zero as cadernetas de poupança ou os títulos federais [...], LFT (Letras Financeiras do Tesouro) e outros, visto que, em cada ocasião, temos um tipo de título como representativo de risco zero.

As principais premissas e a lógica do modelo de Residual Income Valuation proposto por Ohlson (1995) são destacados a seguir. A princípio, (i) o valor da empresa é igual ao valor presente dos seus dividendos esperados e (ii) o PL de um período é igual ao PL do período imediatamente anterior adicionado do resultado contábil do período e diminuído dos dividendos líquidos distribuídos no período, portanto, (iii) os dividendos distribuídos afetam o patrimônio do período mas não afetam o resultado do período (somente os resultados esperados dos períodos seguintes) e, então, (iv) podem ser "substituídos" pelo valor de mercado em uma relação de 1 para 1 , tornando (v) a estratégia de distribuição de dividendos irrelevante para calcular o valor (preço) da empresa, que (vi) passa a ser uma função dos resultados anormais futuros, conforme fórmula abaixo (LOPES, 2001, p. 51-7),

$$
P_{i j}=B V_{i j}+\sum_{t=1}^{\infty} \frac{E_{j}\left[A b_{i j+t}\right]}{(1+r)^{t}}
$$

Assumindo que $\mathrm{E}_{\boldsymbol{j}}\left[\boldsymbol{B} \boldsymbol{V}_{j+t}\right] /(\boldsymbol{1}+\boldsymbol{r})^{t} \rightarrow 0$ quando $\mathrm{t} \mathrm{t} \rightarrow \infty$

Onde:

$\boldsymbol{P}_{i j}$ é o preço das ações da empresa i ao final do período j

$\boldsymbol{B} \boldsymbol{V}_{i j}$ é o Valor Patrimonial (PL) por ação da empresa i ao final do período j

$\boldsymbol{A b}_{i j+t}$ é o Resultado anormal contábil (conforme expresso anteriormente) por ação da empresa i para os períodos $\mathrm{j}+1, \mathrm{j}+2, \ldots, \mathrm{j}+\mathrm{t}$

$\boldsymbol{r}_{j}$ [ ] é o operador matemático do valor esperado condicionado à informaçãopossuída na data $\mathrm{j}$

$r$ é a Taxa de juros livre de risco

Neste modelo então, os resultados anormais passam a ser a base para se valorizar a empresa, ao invés da utilização dos dividendos distribuídos (fluxo de caixa futuro de dividendos distribuídos trazido a valor presente), como a maior parte dos modelos de avaliação de empresa propõem.

\section{PRICE/BOOK ( P/B )}

A relação (ratio) entre o valor de mercado de uma empresa e seu valor patrimonial (valor de livro) é comumente reportada na literatura como Price/Book (quando se estuda os valores unitários das ações das empresas) ou como Market-to-Book (MTB ou ME/BE).

Em alguns casos também se encontra o termo Book-to-Market (BTM ou BE/ME) e este nada mais é do que a razão inversa da expressão anterior (Market-to-Book). Dependendo do que se pretende estudar, adota-se um ou outro: geralmente adota-se aquele para com o qual se espera uma relação direta, facilitando o entendimento das premissas, dos testes e dos resultados.

Outro indicador (ou ratio) muito utilizado e que também relaciona dados de mercado e dados contábeis é o Price/Earnings, relação entre preço da ação e lucro contábil, que até o início da década de noventa foi mais estudado do que a razão entre os valores de mercado e de patrimômio da empresa, segundo Penman (1996, p. 235):

The P/B ratio received less academic attention until Fama and French [1992]. Traditionally, it was interpreted as indicating expected return on equity (see, for example, Graham, Dodd, and Cottle [1962]). This interpretation is given expression in the standard formula reconciling price to book value (in Preinreich [1938], Edwards and Bell [1961], and Peasnell [1982], for example). Like P/E, P/B has been modeled as a growth indicator (Preinreich [1932], kay [1976], and Brief and Lawson [1992]. (...) Like P/E, it has been suggested as an indicator of mispriced stocks (Rosenberg, Reid, and Lanstein [1985]) and of a 'value stock' versus a 'glamour stock' (Lakonishok, 
Shleifer, and Vishny [1994]). Recent work by Chan, Hamao, and Lakonishok [1991] and Fama and French [1992] has documented that the P/B ratio explains mean stock returns, accompanied by conjectures that it is a proxy for risk or an indicator of distress, the difference between market and book leverage, or mispriced stocks.

Fama e French (1995, p. 131 e 139) ressaltaram que

"it is no surprise that book-to-market-equity is associated with persistent properties of earnings. (...) Consistent with rational pricing, high BE/ME signals persistent poor earnings and low BE/ME signals strong earnings. (...) but asset pricing need not to be rational. With irrational pricing, stocks prices and BE/ME can be driven by swings in investor sentiment that have little to do with long-term earnings".

Fama e French (1995, p. 142) também salientaram que aconteceria uma precificação irracional dos ativos caso o mercado não compreendesse a natureza do crescimento dos lucros:

The market incorrectly extrapoles the strong earnings growth of low-BE/ME stocks and the weak growth of high-BE/ME stocks. Low-BE/ME stocks then have low average returns after portfolio formation because their earnings growth is weaker than the market expects, and high-BE/ME stocks have high average returns because their earnings growth is stronger than expected.

\section{HIPÓTESE}

Partindo-se de uma racional precificação dos ativos pelo mercado, supõe-se que aquelas ações mais valorizadas pelo mercado em relação ao valor patrimonial da empresa, ou seja, as que apresentam uma maior relação Price/Book, sejam aquelas empresas que gerem maiores resultados anormais, já que, segundo o Modelo de Residual Income Valuation de Ohlson e suas premissas, os resultados anormais são a base para se calcular o valor da empresa e o preço de suas ações.

Testou-se a seguinte hipótese para verificar se isto pode estar ocorrendo com as empresas no mercado brasileiro, a partir das empresas listadas na Bovespa:

\section{$H_{0}$ - As ações negociadas na Bolsa de Valores de São Paulo que possuem os maiores Price/Book são as das empresas que apresentam os maiores resultados anormais.}

Para se testar esta hipótese foi feito um estudo de carteiras, onde foi segregada uma carteira/quartil de ações com os maiores Price/Book e outra com os menores Price/Book e analisou-se se aquela apresentava maiores resultados anormais do que esta última, a um nível de significância de 5\%, conforme metodologia descrita a seguir.

\section{DADOS E METODOLOGIA}

As amostras (anuais) deste estudo foram extraídas do banco de dados da Economática, empresa especializada em informações para o mercado de capitais. Os dados coletados referem-se às empresas que têm (ou tiveram) ações negociadas na Bolsa de Valores de São Paulo durante o período de 1996 a 2014 e constituemse principalmente de valores de Preço/Valor Patrimonial por Ação (Price/Book); Lucro por Ação; Patrimônio Líquido por Ação (referente a 1995 inclusive); Preço da Ação, e Volumes Negociados por Ação, todos relativos ao final de cada ano fiscal (31 de dezembro de cada ano, no caso do Brasil). Limitou-se o escopo do estudo ao período compreendido entre anos de 1996 a 2014 devido à maior disponibilidade dos dados bem como à relativa estabilidade econômica brasileira após o Plano Real.

Foram selecionados dados de todas as empresas e respectivas ações ordinárias e/ou preferenciais, negociadas na BOVESPA, de todos os setores da economia, desde que negociadas por pelo menos dois anos seguidos (para que se pudesse efetuar cálculos que necessitassem de dados do ano anterior). Ou seja, não foi 
exigido que as empresas apresentassem dados para todos os anos analisados: uma empresa pode fazer parte da amostra em determinado(s) ano(s) e não fazer parte em outro(s) ano(s).

Não exigindo a participação das empresas em todas as amostras de 1996 a 2014 evita-se o chamado viés de sobrevivência (survivor bias), pois de outro modo somente as empresas mais "fortes", listadas na Bolsa há mais tempo e que "sobreviveram" até 2014 seriam consideradas no estudo, o que poderia levar a resultados enviesados (tendenciosos).

Foram excluídas na amostra de determinado ano as ações daquelas empresas cujo Patrimônio Líquido estivesse negativo ou no ano imediatamente anterior ou ao final do ano em questão, seguindo a linha de Fama e French (1995, p. 138) que também excluíram ações com PL negativo de suas amostras. Quando as empresas apresentavam dados de várias ações ordinárias e/ou preferenciais, foram eliminadas as ações com menos cotações/ negociações, mantendo-se na amostra anual somente uma ação por empresa a cada ano considerado.

Foram extraídas diretamente da Economática, as relações de Price/Book ratio (Preço/Valor Patrimonial da Ação, ou P/VPA) e dados de Lucro por Ação (LPA) ao final de cada ano, além do Patrimônio Líquido por Ação (Valor Patrimonial por Ação, ou VPA) relativo ao final do ano imediatamente anterior. Aplicou-se a taxa de juros livre de risco (índice anual da poupança, neste estudo) sobre o VPA do ano anterior e o produto obtido foi então subtraído do Resultado Contábil por Ação (LPA) do ano em questão, apurando-se assim o Resultado Anormal por Ação, de acordo com a fórmula expressa na seção dois deste trabalho. Para que estes Resultados Anormais por Ação (RAA) calculados fossem apropriadamente comparáveis, os mesmos foram apresentados em percentuais sobre o PL (mesma base, portanto, sobre a qual foram calculados). Procedendo-se desta forma evitam-se o efeito escala (conforme sugerido por Brown et al, 1999) e o efeito tamanho (size effect), pois uma grande empresa, por exemplo, pode auferir um resultado anormal monetariamente maior que uma pequena empresa e, no entanto, ter uma lucratividade relativa menor que a da segunda.

Após estes filtros, premissas e cálculos, cada amostra anual (dados ano a ano), caso a caso, foi analisada para se verificar a existência de outliers. Optou-se por um corte (trimming) de 5\% (cinco por cento) em cada amostra anual para a eliminação de outliers. Vale ressaltar que com isso o teste se tornou, em geral, mais robusto, pois as médias das carteiras a serem formadas ficaram menos distantes umas das outras e com menos chances de serem estatisticamente diferentes. A Tabela 1 apresenta a Estatística Descritiva das variáveis após este processo.

Tabela 1 - Estatística Descritiva da Amostra Valores de Price/Book ( P/B ou P/VPA) e Resultados Anormais por Ação

\begin{tabular}{ccccccc}
\hline Variáveis & N & Média & Desvio Padrão & Mediana & Máximo & Mínimo \\
\hline P / B (1996) & 200 & 0,69 & 0,61 & 0,5 & 4,0 & 0,1 \\
\hline P / B (1997) & 188 & 0,74 & 0,63 & 0,5 & 3,6 & 0,1 \\
\hline P / B (1998) & 180 & 0,48 & 0,35 & 0,4 & 2,0 & 0,1 \\
\hline P / B (1999) & 244 & 1,00 & 0,92 & 0,7 & 5,8 & 0,1 \\
\hline P / B (2000) & 224 & 0,97 & 0,94 & 0,7 & 5,9 & 0,1 \\
\hline P / B (2001) & 228 & 0,94 & 1,00 & 0,7 & 8,9 & 0,1 \\
\hline P / B (2002) & 208 & 0,95 & 1,06 & 0,7 & 9,4 & 0,1 \\
\hline P / B (2003) & 208 & 1,28 & 0,99 & 1,0 & 5,8 & 0,2 \\
\hline P / B (2004) & 212 & 1,68 & 1,45 & 1,2 & 9,0 & 0,1 \\
\hline P / B (2005) & 204 & 1,88 & 1,82 & 1,4 & 10,2 & 0,2 \\
\hline P / B (2006) & 204 & 2,27 & 1,81 & 2,0 & 11,4 & 0,2 \\
\hline P / B (2007) & 252 & 2,86 & 2,43 & 2,3 & 20,3 & 0,3 \\
\hline P / B (2008) & 244 & 1,47 & 1,71 & 1,0 & 15,5 & 0,2 \\
\hline P / B (2009) & 248 & 2,23 & 1,91 & 1,7 & 15,2 & 0,3 \\
\hline P / B (2010) & 252 & 2,10 & 1,88 & 1,6 & 15,4 & 0,1 \\
\hline P / B (2011) & 252 & 1,74 & 1,49 & 1,3 & 9,4 & 0,3 \\
\hline P / B (2012) & 260 & 1,92 & 1,69 & 1,4 & 10,4 & 0,2 \\
\hline P / B (2013) & 260 & 1,81 & 1,55 & 1,2 & 9,9 & 0,2 \\
\hline P / B (2014) & 248 & 1,68 & 1,74 & 1,1 & 11,7 & 0,2 \\
\hline
\end{tabular}


Tabela 1 - Estatística Descritiva da Amostra Valores de Price/Book ( P/B ou P/VPA) e Resultados Anormais por Ação (continuação)

\begin{tabular}{ccccccc}
\hline Variáveis & N & Média & Desvio Padrão & Mediana & Máximo & Mínimo \\
\hline Result. Anormal (1996) & 200 & $-16 \%$ & $20 \%$ & $-12 \%$ & $27 \%$ & $-100 \%$ \\
\hline Result. Anormal (1997) & 188 & $-12 \%$ & $24 \%$ & $-8 \%$ & $92 \%$ & $-88 \%$ \\
\hline Result. Anormal (1998) & 180 & $-11 \%$ & $19 \%$ & $-8 \%$ & $28 \%$ & $-76 \%$ \\
\hline Result. Anormal (1999) & 244 & $-12 \%$ & $22 \%$ & $-8 \%$ & $38 \%$ & $-105 \%$ \\
\hline Result. Anormal (2000) & 224 & $-4 \%$ & $20 \%$ & $-1 \%$ & $48 \%$ & $-88 \%$ \\
\hline Result. Anormal (2001) & 228 & $-2 \%$ & $21 \%$ & $0 \%$ & $47 \%$ & $-112 \%$ \\
\hline Result. Anormal (2002) & 208 & $-7 \%$ & $26 \%$ & $-3 \%$ & $51 \%$ & $-115 \%$ \\
\hline Result. Anormal (2003) & 208 & $4 \%$ & $25 \%$ & $1 \%$ & $89 \%$ & $-78 \%$ \\
\hline Result. Anormal (2004) & 212 & $9 \%$ & $24 \%$ & $8 \%$ & $99 \%$ & $-89 \%$ \\
\hline Result. Anormal (2005) & 204 & $7 \%$ & $24 \%$ & $5 \%$ & $110 \%$ & $-93 \%$ \\
\hline Result. Anormal (2006) & 204 & $7 \%$ & $19 \%$ & $6 \%$ & $66 \%$ & $-51 \%$ \\
\hline Result. Anormal (2007) & 252 & $8 \%$ & $20 \%$ & $7 \%$ & $67 \%$ & $-82 \%$ \\
\hline Result. Anormal (2008) & 244 & $3 \%$ & $22 \%$ & $3 \%$ & $69 \%$ & $-71 \%$ \\
\hline Result. Anormal (2009) & 248 & $11 \%$ & $30 \%$ & $6 \%$ & $336 \%$ & $-28 \%$ \\
\hline Result. Anormal (2010) & 252 & $10 \%$ & $20 \%$ & $8 \%$ & $140 \%$ & $-39 \%$ \\
\hline Result. Anormal (2011) & 252 & $4 \%$ & $16 \%$ & $3 \%$ & $64 \%$ & $-44 \%$ \\
\hline Result. Anormal (2012) & 260 & $1 \%$ & $19 \%$ & $1 \%$ & $93 \%$ & $-77 \%$ \\
\hline Result. Anormal (2013) & 260 & $2 \%$ & $21 \%$ & $2 \%$ & $111 \%$ & $-78 \%$ \\
\hline Result. Anormal (2014) & 248 & $0 \%$ & $21 \%$ & $1 \%$ & $81 \%$ & $-86 \%$ \\
\hline
\end{tabular}

O passo seguinte foi o processo de formação das carteiras a partir das amostras de cada ano. A formação das carteiras é fundamental para se testar a hipótese deste estudo. A chave para a formação das carteiras foi o indicador Price/Book (P/B), ou a razão entre as variáveis Preço/Valor Patrimonial da Ação (P/VPA). A partir da relação P/B do final de cada ano, as ações de cada amostra anual foram classificadas em ordem crescente de valor. A amostra foi então segmentada em quatro partes/carteiras compostas por $25 \%$ das ações de cada ano, sendo duas delas separadas para a realização dos testes: a Carteira Q1, composta pelas ações posicionadas até o $1^{\circ}$ quartil, e a Carteira Q4, composta pelas ações posicionadas acima do $3^{\circ}$ quartil.

Seguindo trabalhos similares na literatura de finanças e, devido ao reduzido tamanho relativo do mercado de capitais no Brasil, adotou-se a segmentação das amostras anuais em quartis, pois a segmentação por decis poderia levar a amostras pequenas e com pouca representatividade. Penman $(1996)$ e Fama e French $(1992,1995,1996)$ utilizaram-se da mesma metodologia de formação de carteiras, optando ora por decis, ora por quartis. Vale ressaltar que com isso o teste se tornou, em geral, mais robusto, pois as carteiras formadas ficaram menos distantes umas das outras e com menos chances de serem estatisticamente diferentes.

Este processo de formação de carteiras foi feito para cada amostra anual, com base na posição do final de cada ano fiscal, possibilitando a mudança das ações que compunham as carteiras Q1 e Q4 de cada ano (e mesmo da quantidade de ações que compunham as mesmas), de acordo com a variação e ordenamento crescente da razão $\mathrm{P} / \mathrm{B}$ de cada amostra anual.

Com os Resultados Anormais por Ação para as Carteiras Q1 e Q4, ano a ano, foram realizados Testes T para diferença entre as médias para comprovar se os resultados anormais médios encontrados não seriam iguais estatisticamente (a um nível de significância de 5\%). Se as médias forem estatisticamente iguais, não se pode afirmar que os resultados de uma carteira sejam maiores (ou menores) do que os resultados da outra.

Optou-se por aplicar os Testes T para diferença entre médias devido aos mesmos proporcionarem maior robustez aos resultados deste estudo, bem como apresentarem menores restrições em relação ao tamanho das amostras. $\mathrm{O}$ Teste $\mathrm{T}$ pode ser utilizado tanto para amostras pequenas (abaixo de 30 elementos, sem a necessidade dos dados apresentarem a forma da "curva normal"), quanto para amostras maiores, quando seus resultados se assemelham aos resultados do Teste Z para diferenças entre médias (que exige a "normalidade" dos dados amostrais). Os resultados dos testes são apresentados na próxima seção. 


\section{RESULTADOS EMPÍRICOS}

Os resultados apurados imediatamente após a formação das carteiras Q1 e Q4 para cada ano expressam que, para todos os anos, os Resultados Anormais apresentados pelas Carteiras Q4 foram maiores algebricamente do que os Resultados Anormais apresentados pelas Carteiras Q1, como se pode verificar na tabela 2. Ou seja, até então não se pode rejeitar a hipótese deste trabalho de que as empresas que apresentam maior Price/Book são as que apresentam os maiores Resultados Anormais.

Tabela 2 - Estatística Descritiva das Carteiras Q1 e Q4, ano a ano

\begin{tabular}{|c|c|c|c|c|c|c|c|}
\hline Variáveis por Carteira & Ano & $\mathbf{N}$ & Média & $\begin{array}{l}\text { Desvio } \\
\text { Padrão }\end{array}$ & Mediana & Máximo & Mínimo \\
\hline ME / BE da Carteira Q1 & 1996 & 50 & 0,19 & 0,06 & 0,20 & 0,30 & 0,10 \\
\hline ME / BE da Carteira Q4 & 1996 & 50 & 1,48 & 0,71 & 1,20 & 4,00 & 0,90 \\
\hline R.A. (\%) da Carteira Q1 & 1996 & 50 & $-28 \%$ & $17 \%$ & $-24 \%$ & $-9 \%$ & $-76 \%$ \\
\hline R.A. (\%) da Carteira Q4 & 1996 & 50 & $-8 \%$ & $23 \%$ & $-6 \%$ & $27 \%$ & $-98 \%$ \\
\hline ME / BE da Carteira Q1 & 1997 & 47 & 0,21 & 0,08 & 0,20 & 0,30 & 0,10 \\
\hline ME / BE da Carteira Q4 & 1997 & 47 & 1,60 & 0,68 & 1,40 & 3,60 & 0,90 \\
\hline R.A. (\%) da Carteira Q1 & 1997 & 47 & $-19 \%$ & $22 \%$ & $-15 \%$ & $92 \%$ & $-68 \%$ \\
\hline R.A. (\%) da Carteira Q4 & 1997 & 47 & $-9 \%$ & $26 \%$ & $-2 \%$ & $20 \%$ & $-86 \%$ \\
\hline ME / BE da Carteira Q1 & 1998 & 45 & 0,15 & 0,05 & 0,20 & 0,20 & 0,10 \\
\hline ME / BE da Carteira Q4 & 1998 & 45 & 0,95 & 0,37 & 0,80 & 2,00 & 0,60 \\
\hline R.A. (\%) da Carteira Q1 & 1998 & 45 & $-19 \%$ & $15 \%$ & $-15 \%$ & $1 \%$ & $-76 \%$ \\
\hline R.A. (\%) da Carteira Q4 & 1998 & 45 & $-5 \%$ & $21 \%$ & $0 \%$ & $28 \%$ & $-68 \%$ \\
\hline ME / BE da Carteira Q1 & 1999 & 61 & 0,28 & 0,11 & 0,30 & 0,40 & 0,10 \\
\hline ME / BE da Carteira Q4 & 1999 & 61 & 2,25 & 1,04 & 1,90 & 5,80 & 1,20 \\
\hline R.A. (\%) da Carteira Q1 & 1999 & 61 & $-17 \%$ & $15 \%$ & $-12 \%$ & $14 \%$ & $-61 \%$ \\
\hline R.A. (\%) da Carteira Q4 & 1999 & 61 & $-12 \%$ & $28 \%$ & $-4 \%$ & $38 \%$ & $-105 \%$ \\
\hline ME / BE da Carteira Q1 & 2000 & 56 & 0,29 & 0,10 & 0,30 & 0,40 & 0,10 \\
\hline ME / BE da Carteira Q4 & 2000 & 56 & 2,17 & 1,19 & 1,70 & 5,90 & 1,10 \\
\hline R.A. (\%) da Carteira Q1 & 2000 & 56 & $-9 \%$ & $18 \%$ & $-6 \%$ & $25 \%$ & $-88 \%$ \\
\hline R.A. (\%) da Carteira Q4 & 2000 & 56 & $-2 \%$ & $27 \%$ & $4 \%$ & $48 \%$ & $-75 \%$ \\
\hline ME / BE da Carteira Q1 & 2001 & 57 & 0,27 & 0,10 & 0,30 & 0,40 & 0,10 \\
\hline ME / BE da Carteira Q4 & 2001 & 57 & 2,06 & 1,44 & 1,60 & 8,90 & 1,20 \\
\hline R.A. (\%) da Carteira Q1 & 2001 & 57 & $-9 \%$ & $15 \%$ & $-6 \%$ & $17 \%$ & $-55 \%$ \\
\hline R.A. (\%) da Carteira Q4 & 2001 & 57 & $2 \%$ & $29 \%$ & $6 \%$ & $47 \%$ & $-112 \%$ \\
\hline ME / BE da Carteira Q1 & 2002 & 52 & 0,26 & 0,10 & 0,30 & 0,40 & 0,10 \\
\hline ME / BE da Carteira Q4 & 2002 & 52 & 2,15 & 1,54 & 1,60 & 9,40 & 1,10 \\
\hline R.A. (\%) da Carteira Q1 & 2002 & 52 & $-11 \%$ & $16 \%$ & $-8 \%$ & $51 \%$ & $-45 \%$ \\
\hline R.A. (\%) da Carteira Q4 & 2002 & 52 & $-3 \%$ & $34 \%$ & $7 \%$ & $39 \%$ & $-115 \%$ \\
\hline ME / BE da Carteira Q1 & 2003 & 52 & 0,40 & 0,13 & 0,40 & 0,60 & 0,20 \\
\hline ME / BE da Carteira Q4 & 2003 & 52 & 2,61 & 1,05 & 2,20 & 5,80 & 1,70 \\
\hline R.A. (\%) da Carteira Q1 & 2003 & 52 & $-9 \%$ & $18 \%$ & $-7 \%$ & $48 \%$ & $-64 \%$ \\
\hline R.A. (\%) da Carteira Q4 & 2003 & 52 & $17 \%$ & $29 \%$ & $20 \%$ & $81 \%$ & $-60 \%$ \\
\hline ME / BE da Carteira Q1 & 2004 & 53 & 0,51 & 0,17 & 0,50 & 0,80 & 0,10 \\
\hline ME / BE da Carteira Q4 & 2004 & 53 & 3,60 & 1,66 & 2,90 & 9,00 & 2,10 \\
\hline R.A. (\%) da Carteira Q1 & 2004 & 53 & $-4 \%$ & $18 \%$ & $-4 \%$ & $89 \%$ & $-62 \%$ \\
\hline R.A. (\%) da Carteira Q4 & 2004 & 53 & $21 \%$ & $29 \%$ & $22 \%$ & $99 \%$ & $-89 \%$ \\
\hline
\end{tabular}


Tabela 2 - Estatística Descritiva das Carteiras Q1 e Q4, ano a ano (continuação)

\begin{tabular}{|c|c|c|c|c|c|c|c|}
\hline Variáveis por Carteira & Ano & $\mathbf{N}$ & Média & $\begin{array}{l}\text { Desvio } \\
\text { Padrão }\end{array}$ & Mediana & Máximo & Mínimo \\
\hline ME / BE da Carteira Q1 & 2005 & 51 & 0,46 & 0,16 & 0,50 & 0,70 & 0,20 \\
\hline ME / BE da Carteira Q4 & 2005 & 51 & 4,28 & 2,15 & 3,40 & 10,20 & 2,30 \\
\hline R.A. (\%) da Carteira Q1 & 2005 & 51 & $-4 \%$ & $15 \%$ & $-4 \%$ & $34 \%$ & $-74 \%$ \\
\hline R.A. (\%) da Carteira Q4 & 2005 & 51 & $20 \%$ & $29 \%$ & $20 \%$ & $110 \%$ & $-66 \%$ \\
\hline ME / BE da Carteira Q1 & 2006 & 51 & 0,59 & 0,19 & 0,60 & 0,90 & 0,20 \\
\hline ME / BE da Carteira Q4 & 2006 & 51 & 4,66 & 1,91 & 3,90 & 11,40 & 3,00 \\
\hline R.A. (\%) da Carteira Q1 & 2006 & 51 & $-7 \%$ & $13 \%$ & $-5 \%$ & $30 \%$ & $-51 \%$ \\
\hline R.A. (\%) da Carteira Q4 & 2006 & 51 & $17 \%$ & $24 \%$ & $17 \%$ & $62 \%$ & $-51 \%$ \\
\hline ME / BE da Carteira Q1 & 2007 & 63 & 0,98 & 0,32 & 1,00 & 1,50 & 0,30 \\
\hline ME / BE da Carteira Q4 & 2007 & 63 & 5,81 & 3,22 & 4,40 & 20,30 & 3,30 \\
\hline R.A. (\%) da Carteira Q1 & 2007 & 63 & $-2 \%$ & $17 \%$ & $-2 \%$ & $50 \%$ & $-65 \%$ \\
\hline R.A. (\%) da Carteira Q4 & 2007 & 63 & $15 \%$ & $23 \%$ & $16 \%$ & $65 \%$ & $-82 \%$ \\
\hline ME / BE da Carteira Q1 & 2008 & 61 & 0,43 & 0,13 & 0,40 & 0,60 & 0,20 \\
\hline ME / BE da Carteira Q4 & 2008 & 61 & 3,27 & 2,62 & 2,40 & 15,50 & 1,70 \\
\hline R.A. (\%) da Carteira Q1 & 2008 & 61 & $-3 \%$ & $15 \%$ & $0 \%$ & $49 \%$ & $-49 \%$ \\
\hline R.A. (\%) da Carteira Q4 & 2008 & 61 & $7 \%$ & $28 \%$ & $10 \%$ & $69 \%$ & $-71 \%$ \\
\hline ME / BE da Carteira Q1 & 2009 & 62 & 0,79 & 0,21 & 0,80 & 1,10 & 0,30 \\
\hline ME / BE da Carteira Q4 & 2009 & 62 & 4,61 & 2,44 & 3,60 & 15,20 & 2,70 \\
\hline R.A. (\%) da Carteira Q1 & 2009 & 62 & $0 \%$ & $12 \%$ & $-1 \%$ & $51 \%$ & $-26 \%$ \\
\hline R.A. (\%) da Carteira Q4 & 2009 & 62 & $25 \%$ & $53 \%$ & $15 \%$ & $336 \%$ & $-28 \%$ \\
\hline ME / BE da Carteira Q1 & 2010 & 63 & 0,66 & 0,21 & 0,70 & 0,90 & 0,10 \\
\hline ME / BE da Carteira Q4 & 2010 & 63 & 4,49 & 2,36 & 3,80 & 15,40 & 2,60 \\
\hline R.A. (\%) da Carteira Q1 & 2010 & 63 & $1 \%$ & $12 \%$ & $1 \%$ & $48 \%$ & $-27 \%$ \\
\hline R.A. (\%) da Carteira Q4 & 2010 & 63 & $21 \%$ & $27 \%$ & $20 \%$ & $140 \%$ & $-36 \%$ \\
\hline ME / BE da Carteira Q1 & 2011 & 63 & 0,57 & 0,14 & 0,60 & 0,80 & 0,30 \\
\hline ME / BE da Carteira Q4 & 2011 & 63 & 3,75 & 1,69 & 3,00 & 9,40 & 2,20 \\
\hline R.A. (\%) da Carteira Q1 & 2011 & 63 & $-6 \%$ & $13 \%$ & $-3 \%$ & $42 \%$ & $-44 \%$ \\
\hline R.A. (\%) da Carteira Q4 & 2011 & 63 & $15 \%$ & $18 \%$ & $14 \%$ & $54 \%$ & $-35 \%$ \\
\hline ME / BE da Carteira Q1 & 2012 & 65 & 0,56 & 0,19 & 0,60 & 0,80 & 0,20 \\
\hline ME / BE da Carteira Q4 & 2012 & 65 & 4,22 & 1,83 & 3,70 & 10,40 & 2,40 \\
\hline R.A. (\%) da Carteira Q1 & 2012 & 65 & $-8 \%$ & $11 \%$ & $-6 \%$ & $15 \%$ & $-51 \%$ \\
\hline R.A. (\%) da Carteira Q4 & 2012 & 65 & $8 \%$ & $27 \%$ & $12 \%$ & $93 \%$ & $-77 \%$ \\
\hline ME / BE da Carteira Q1 & 2013 & 65 & 0,53 & 0,18 & 0,60 & 0,80 & 0,20 \\
\hline ME / BE da Carteira Q4 & 2013 & 65 & 3,98 & 1,55 & 3,70 & 9,90 & 2,40 \\
\hline R.A. (\%) da Carteira Q1 & 2013 & 65 & $-9 \%$ & $17 \%$ & $-5 \%$ & $28 \%$ & $-67 \%$ \\
\hline R.A. (\%) da Carteira Q4 & 2013 & 65 & $11 \%$ & $28 \%$ & $14 \%$ & $111 \%$ & $-75 \%$ \\
\hline ME / BE da Carteira Q1 & 2014 & 62 & 0,41 & 0,14 & 0,40 & 0,60 & 0,20 \\
\hline ME / BE da Carteira Q4 & 2014 & 62 & 3,97 & 2,10 & 3,25 & 11,70 & 1,90 \\
\hline R.A. (\%) da Carteira Q1 & 2014 & 62 & $-12 \%$ & $17 \%$ & $-7 \%$ & $17 \%$ & $-83 \%$ \\
\hline R.A. (\%) da Carteira Q4 & 2014 & 62 & $13 \%$ & $27 \%$ & $16 \%$ & $81 \%$ & $-86 \%$ \\
\hline
\end{tabular}

Entretanto, após a realização dos testes para apurar se os Resultados Anormais médios das Carteiras Q1 e Q4 de cada amostra anual seriam diferentes estatisticamente, pode-se constatar, na tabela 3, que em três anos/ amostras (1999, 2000 e 2002), de um total de dezenove testados, os Resultados Anormais foram considerados estatisticamente iguais considerando-se um nível de confiança de 95\%.

Portanto, para estes três anos/amostras não se pode afirmar que os Resultados Anormais das Carteiras Q4 sejam maiores que os Resultados Anormais das Carteiras Q1, a um nível de confiança de 95\%. 
Tabela 3 - Resultado dos Testes para Diferença entre Médias dos Resultados Anormais das Carteiras Q1 e

\begin{tabular}{|c|c|c|c|c|c|c|c|c|}
\hline \multicolumn{9}{|c|}{ Q4, ano a ano } \\
\hline \multirow{2}{*}{ Ano } & R.A. (\%) & Médio & \multirow{2}{*}{$\begin{array}{c}\text { médias estatist. } \\
\text { diferentes? }\end{array}$} & \multirow{2}{*}{$\begin{array}{l}\text { p-value } \\
\text { bicaudal }\end{array}$} & \multirow{2}{*}{$\begin{array}{c}\text { p-value } \\
\text { unicaudal }\end{array}$} & \multirow{2}{*}{ stat t } & \multirow{2}{*}{$\begin{array}{l}\text { t crítico } \\
\text { bicaudal }\end{array}$} & \multirow{2}{*}{$\begin{array}{c}\text { t crítico } \\
\text { unicaudal }\end{array}$} \\
\hline & Q1 & Q4 & & & & & & \\
\hline 1996 & $-28 \%$ & $-8 \%$ & $\operatorname{sim}$ & 0,000 & 0,000 & 4,95 & 1,98 & 1,66 \\
\hline 1997 & $-19 \%$ & $-9 \%$ & $\operatorname{sim}$ & 0,038 & 0,019 & 2,11 & 1,99 & 1,66 \\
\hline 1998 & $-19 \%$ & $-5 \%$ & $\operatorname{sim}$ & 0,001 & 0,000 & 3,60 & 1,99 & 1,66 \\
\hline 1999 & $-17 \%$ & $-12 \%$ & Não & 0,197 & 0,098 & 1,30 & 1,98 & 1,66 \\
\hline 2000 & $-9 \%$ & $-2 \%$ & Não & 0,104 & 0,052 & 1,64 & 1,98 & 1,66 \\
\hline 2001 & $-9 \%$ & $2 \%$ & $\operatorname{sim}$ & 0,014 & 0,007 & 2,51 & 1,98 & 1,66 \\
\hline 2002 & $-11 \%$ & $-3 \%$ & Não & 0,130 & 0,065 & 1,53 & 1,98 & 1,66 \\
\hline 2003 & $-9 \%$ & $17 \%$ & $\operatorname{sim}$ & 0,000 & 0,000 & 5,43 & 1,98 & 1,66 \\
\hline 2004 & $-4 \%$ & $21 \%$ & $\operatorname{sim}$ & 0,000 & 0,000 & 5,26 & 1,98 & 1,66 \\
\hline 2005 & $-4 \%$ & $20 \%$ & $\operatorname{sim}$ & 0,000 & 0,000 & 5,43 & 1,98 & 1,66 \\
\hline 2006 & $-7 \%$ & $17 \%$ & $\operatorname{sim}$ & 0,000 & 0,000 & 6,33 & 1,98 & 1,66 \\
\hline 2007 & $-2 \%$ & $15 \%$ & $\operatorname{sim}$ & 0,000 & 0,000 & 4,83 & 1,98 & 1,66 \\
\hline 2008 & $-3 \%$ & $7 \%$ & $\operatorname{sim}$ & 0,015 & 0,008 & 2,46 & 1,98 & 1,66 \\
\hline 2009 & $0 \%$ & $25 \%$ & $\operatorname{sim}$ & 0,000 & 0,000 & 3,61 & 1,98 & 1,66 \\
\hline 2010 & $1 \%$ & $21 \%$ & $\operatorname{sim}$ & 0,000 & 0,000 & 5,14 & 1,98 & 1,66 \\
\hline 2011 & $-6 \%$ & $15 \%$ & $\operatorname{sim}$ & 0,000 & 0,000 & 7,32 & 1,98 & 1,66 \\
\hline 2012 & $-8 \%$ & $8 \%$ & $\operatorname{sim}$ & 0,000 & 0,000 & 4,44 & 1,98 & 1,66 \\
\hline 2013 & $-9 \%$ & $11 \%$ & $\operatorname{sim}$ & 0,000 & 0,000 & 4,85 & 1,98 & 1,66 \\
\hline 2014 & $-12 \%$ & $13 \%$ & $\operatorname{sim}$ & 0,000 & 0,000 & 6,13 & 1,98 & 1,66 \\
\hline
\end{tabular}

Os resultados destes testes indicam, portanto, que se pode aceitar a hipótese deste trabalho para dezesseis dos dezenove anos pesquisados. No entanto, para três anos não se pode aceitar esta mesma hipótese, já que para estes anos não se pode comprovar que os Resultados Anormais das carteiras Q4 sejam estatisticamente maiores que os das carteiras Q1, a um nível de significância de cinco por cento.

Optou-se por reportar tanto os resultados bicaudais quanto os unicaudais dos testes $\mathrm{T}$ a fim de deixar aos usuários da informação a avaliação quanto a utilidade da informação. Os testes bicaudais testam a diferença entre médias para os dois lados/caudas da distribuição (possuindo portanto duas áreas de rejeição para a hipótese nula de igualdade entre as médias) enquanto que os testes unicaudais testam a diferença entre médias para somente um dos lados/caudas da distribuição (possuindo portanto somente uma área de rejeição para a hipótese nula de igualdade entre as médias), sendo mais utilizada para se testar somente se uma média é maior (ou menor) que a outra. Os resultados dos testes unicaudais e bicaudais sinalizam para a mesma direção, entretanto, dependendo do objetivo, pode-se optar por um ou por outro, dependendo de premissas que devem se originar de fora da estatística.

Portanto neste estudo, os resultados do p-value unicaudal recusam a hipótese de que a média de Q4 seja maior que Q1 a um nível de confiança de 95\% para os anos de 1999 ( $p$-value $=0,098), 2000(p$-value $=0,052)$ e 2002 ( $p$-value $=0,065)$. Entretanto já não recusariam a um nível de confiança de $90 \%$.

Já os resultados do $p$-value bicaudal recusam a hipótese de que a média de Q4 seja diferente (maior ou menor) que Q1 a um nível de confiança de 95\% para os anos de 1999 (p-value = 0,197), 2000 ( $p$-value $=0,104)$ e 2002 ( $p$-value $=0,130)$. Seriam recusados também a um nível de confiança de $90 \%$ e somente não seriam recusados a um nível de confiança de $80 \%$.

\section{CONSIDERAÇÕES FINAIS}

Os resultados dos testes empíricos para dezesseis dos dezenove anos pesquisados são estatisticamente significativos a um nível de $95 \%$ de confiança e, embora os resultados de três períodos (1999, 2000 e 2002) 
apontem para a rejeição da hipótese, vale ressaltar que, dependendo da finalidade ou do modelo decisório do usuário, mesmo os resultados destes três anos também poderiam ser considerados como de grande valia (por exemplo, caso se admitisse um nível de confiança de oitenta por cento, todos os anos apresentariam resultados significantes).

Mesmo com esta rejeição em três anos/amostras, pode-se constatar que modelos de avaliação baseados em números contábeis podem apresentar significativa relevância informacional para o Mercado de Capitais no Brasil, conforme já atestado em outros estudos recentes (LOPES, 2001, 2005; LOPES e GALDI, 2007; SANTANNA, 2004).

Cabe ressaltar também que nos últimos doze anos pesquisados (de 2003 a 2014) todos os resultados foram significativos a um nível de confiança de 95\% (e continuariam significativos mesmo se fosse considerado um nível de confiança de 98\%). Isto pode sugerir que os números contábeis tenham aumentado sua relevância informacional, ou que, simplesmente, não tenham acontecidos distúrbios significativos que pudessem atrapalhar a "boa" precificação dos ativos, como notadamente ocorreu em 1999 e 2002 (anos rejeitados nos testes).

Em 1999, 2000 e 2002 o p-value registrado nos testes foram significativamente maiores que nos outros anos (o p-value bicaudal dos anos de 1999 foi de 0,197, o p-value de 2000 foi de 0,104 e o p-value de 2002 foi de 0,130 enquanto que em nenhum outro ano foi maior do que 0,040 ) e podem ter sido gerados por distúrbios concomitantes nas áreas econômica, política e até normativa/fiscal (caso de 1999).

Em 1999 houve, por exemplo, um distúrbio econômico (maxidesvalorização do Real) que acabou por levar a um outro normativo/fiscal, com a decisão do Fisco de permitir o diferimento desta variação cambial em vários períodos, bem como deixar a cargo de cada empresa se adotaria o diferimento cambial ou não. Em 2000, não houve acontecimentos tão marcantes quanto estes de 1999, a não ser, talvez, o próprio efeito, diluído, do diferimento da desvalorização cambial ainda em vigor. Em 2002 houve outra grande volatilidade/ desvalorização cambial do Real combinada com o efeito político das eleições no Brasil e às grandes incertezas quanto ao governo que assumiria o país em janeiro de 2003.

Estes acontecimentos podem, portanto, ter prejudicado a precificação dos ativos/ações e levado aos resultados apontados na Tabela 3. Uma análise destes fatores e de outros não tão visíveis poderiam muito contribuir para o desenvolvimento da contabilidade no Brasil e para o aumento da relevância dos números contábeis para toda a sociedade.

Vale ressaltar que os testes adotados neste trabalho não permitem fazer inferências mais contundentes quanto ao reflexo da mudança da legislação antes e após a adoção das Normas Internacionais de Contabilidade (IFRS) pela Legislação Societária brasileira. Entretanto, os resultados obtidos sugerem que a adoção do IFRS não produziu distúrbios significativos nos resultados dos testes, já que as últimas 12 amostras/anos apresentaram resultados anormais médios estatisticamente diferentes ao nível de significância de $98 \%$.

\section{REFERÊNCIAS BIBLIOGRÁFICAS}

BALL, R. J. BROWN, W. An empirical evaluation of accounting income numbers. Journal of Accounting Research, Vol. 6, p.159-178, Autumn 1968.

BANZ, Rolf W.; BREEN, William J. Sample-dependent results using accounting and market data: some evidence. The Journal of Finance, v. 41, n. 4, p. 779-793, 1986.

BASU, S. Investment performance of common stocks in relation to their price/earnings ratio: a test of the efficient market hypothesis. The Journal of Finance, v.32, p. 663-682, 1977.

BROWN, Stephen; LO, Kin; LYS, Thomas. Use of R2 in accounting research: measuring changes in value relevance over the last four decades. Journal of Accounting and Economics, New York, v.28, n.I, p.83-115, 1999.

CHAN, L.K.; JEGADEESH, N.; LAKONISHOK, J. Evaluating the performance of value versus glamour stocks: the impact of selection bias. Journal of Financial Economics, v.38, p. 269-296, 1995.FAMA, E.F.; FRENCH, K.R. The cross-section of expected stock returns. The Journal of Finance, v.47, p.427-465, 1992. 
Size and Book-to-Market factors in Earnings and Returns. The Journal of Finance, v.50, n. 1, p.131-55, 1995.

The CAPM is Wanted, Dead or Alive. The Journal of Finance, v.51, n. 5, p.1947-58, 1996.

. Value versus Growth: The internatonal evidence. The Journal of Finance, v.53, p.1975-1998, 1998.

JACOBSEN, Robert. The persistence of abnormal returns. Strategic Management Journal, v. 9, n. 5, p.415-430, 1988.

LAKONISHOK, J.; SHLEIFER, A.; VISHNY, R. W. Contrarian investment, extrapolation, and risk. Journal of Finance, v.49, p.1541-1578, 1994.

LOPES, Alexsandro Broedel. A Relevância da informação contábil para o mercado de capitais: o modelo de Ohlson aplicado à Bovespa. 2001. Tese (Doutorado em Ciências Contábeis) - Departamento de Contabilidade e Atuária - FEA/USP.

Financial accounting in Brazil: an empirical examination. Latin American Business Review, n.6, p. 45-68, 2005.

GALDI, F. Does financial statement analysis generate abnormal returns under extremely adverse conditions? Paper apresentado no Annual Meeting of the American Accounting Association, Chicago, Illinois, 2007.

OHLSON, James. A. Earnings, Book Values and Dividends in Equity Valuation. Contemporary Accounting Research, v.11, n. 2, p. 661-687, 1995.

PENMAN, Stephen H. The Articulation of Price-Earnings Ratios and Market-to-Book Ratios and the Evalution of Growth. Journal of Accounting Research, v.34, n. 2, p. 235-59, 1996.

ROSS, Stephen A., WESTERFIELD, RANDOLPH W., e JAFFE, JEFFREY F. Administração Financeira: Corporate Finance. São Paulo: Atlas, 1995.

SANT'ANNA, Dimitri P. de. A relevância das informações contábeis na Bovespa: avaliação dos modelos de Residual Income Valuation e Abnormal Earnings Growth. 2004. Dissertação (Mestrado em Ciências Contábeis) - Fundação Instituto Capixaba de Pesquisas em Contabilidade, Economia e Finanças (Fucape), Vitória-ES

SECURATO, J. R. Decisões Financeiras em condições de risco. São Paulo: Atlas, 1996.

STEVENSON, William J. Estatística Aplicada à Administração. São Paulo: Editora Harbra, 1986. 